\title{
Value of information exposed: wireless networking solutions to the parking search problem
}

\author{
Evangelia Kokolaki, Merkouris Karaliopoulos, Ioannis Stavrakakis \\ Department of Informatics and Telecommunications \\ National and Kapodistrian University of Athens, Athens, Greece \\ \{evako, mkaralio, ioannis\}@di.uoa.gr
}

\begin{abstract}
Our work draws on a concrete parking space search application to explore fundamental tradeoffs of wireless networking solutions to the provision of real-life services. In particular, we consider a city area, wherein each vehicle (mobile user) moves towards a chosen destination and seeks vacant parking space in its vicinity. Three main approaches to the parking space search problem are investigated, each representing a distinct paradigm of how wireless networking communications can assist the information management process. In the first approach, the vehicles execute the currently common "blind" sequential search for parking space by wandering around the destination. In the second distributed approach, the vehicles, while moving around the area, opportunistically collect and share with each other information on the location and status of each parking spot they encounter. Finally, with the third approach, the allocation of parking spots is managed by a central server availing global knowledge about the parking space availability.
\end{abstract}

We compare the three approaches with respect to the time and distance vehicles need to travel before they park as well as the proximity of the assigned parking spots to the travel destinations. Results obtained under two scenarios for the user travel preferences (uniformly distributed travel destinations vs. a single hotspot road), reveal that the relative performance of the three solutions can vary significantly and not always inline with intuition. In the hotspot scenario, the centralized system consistently yields the minimum times and distances at the expense of more distant parking spot assignments; whereas, when user travel destinations are uniformly distributed, the relative performance of all three schemes changes as the vehicle volume grows, with the centralized approach gradually becoming the worst solution. We discuss the way each approach modulates the information dissemination process in space and time and resolves the emerging competition for the parking resources. We also outline models for getting analytical insights to the behavior of the centralized approach.

Keywords: vehicular communications, opportunistic dissemination, parking assistance, machine interference problem

\section{INTRODUCTION}

The efficient use of urban space has been always both a requirement and a challenge in the process of city planning. It calls for several interventions in the way cities are organized, including the efficient management of the car volumes that daily visit the city centre and other popular in-city destinations. Part of this task is the effective operation of the sometimes minimal parking space. The reduction of time that vehicles spend searching for parking places does not alleviate only the traffic congestion problems but also the environmental burden.

The real dimensions of the parking place search problem are a function of several factors. The existence of popular destinations, personal parking preferences, and the drivers' unwillingness to park but only in close proximity to the destination, aggravate the problem. Academic research but also public and/or private initiatives have made a remarkable effort in the last years to solve the problem through parkingassistance systems. Common feature of these systems is the exploitation of wireless communications and information sensing technologies to collect and share information about the availability of parking space in the search area. This way, the parking choices of drivers are steered so that the effective competition over the parking space is reduced and the overall search process becomes more efficient.

In this paper, we draw on two basic systems for parkingassistance that represent broader paradigms of how wireless networking communications can assist the information management process. The first one is opportunistic. Vehicles are equipped with sensors, which allow them to sense the location and status of parking spots as they move across the city area. This information is subsequently shared upon encounters with other vehicles, using medium-range radio communication technologies such as $802.11 \mathrm{x}$. The second system we study is centralized. This time a central server collects both the requests of vehicles for parking space allocation and information about the status of individual parking spots via longer-range radio technologies such as cellular radio. With this information at hand, it selects and reserves parking places for the vehicles, and directs them thereto.

We carry out a systematic simulation study that compares the two radically different paradigms for parking assistance: a) with each other; b) against the current-practice non-assisted system, where vehicles wander in the area around their travel destination searching for parking space. Two scenarios drive our discussion. The first one involves vehicles seeking parking space all over the city area (uniformly distributed destinations). The second scenario features a single area that acts as an attraction pole for vehicles (hot-spot). The effectiveness of the parking search process is captured into three metrics: parking (space) search time and route length, and proximity of the found/assigned parking spot to the user travel destination.

Our results suggest that there is no one-size-fits-all solution; on the contrary, the relative performance of the three 
schemes varies significantly, and not always intuitively, with the operational conditions. In the hotspot scenario, the centralized system consistently yields the minimum times and distances. With global knowledge of parking space availability throughout the area, it can resolve better the competition amongst the vehicles for the few parking places around the common destinations. Assuming that the user is willing to park somehow further than the destination, his search time is significantly reduced. On the contrary, the opportunistic system ends up recycling information that synchronizes the movement patterns of the vehicles and intensifies their competition. Even for moderate number of vehicles, the opportunistic scheme effectively "degenerates" to the non-assisted scheme. When user travel destinations are uniformly distributed, the relative performance of the three schemes changes with the vehicle volume. The initially optimal centralized approach gradually becomes the worst solution since the reservation system ends up wasting part of the parking capacity of the system at high load. The opportunistic scheme ranks top since vehicles exploit the information they collect upon their encounters to make informed searches and reach a vacant parking spot faster.

We describe the three parking approaches in Section II, where we also outline models for the analytical investigation of the centralized approach. The paper proceeds with the definition of the performance metrics and presentation of the simulation environment used for our evaluations in Section III. Section IV presents the evaluation results and discusses the reasons behind them. We outline related work on the subject in Section V and conclude the paper in Section VI with a summary of our results and plans for future work.

\section{Approaches to Parking Space SEARCH}

We summarize three basic approaches to the parking space search problem. Each of them defines a different strategy for localizing and occupying vacant parking spots ${ }^{1}$. In all three cases, there is a fixed set of parking spots $\mathcal{P}$, with $\mid \mathcal{P}=\mathrm{P}$, distributed across a city area $A$, and a finite population of vehicles $C$, with $|C|=\mathrm{C}$ moving therein. At each point in time, a vehicle $\mathrm{c} \in C$ may be in one of three states: parked, on the way towards some user destination $d_{\mathrm{c}}$, or in search of a parking spot. The vehicle exits the second state and enters the third one as soon as it approaches its travel destination, i.e., when it enters the initial parking search area (see Figure 1).

The main differentiation factor among the three approaches (and several variants thereof) is the way users (i.e., vehicles) exploit, or do not exploit, information about the availability of parking space within the search area. Each parking spot is equipped with a sensor providing information about its occupancy status. Vehicles, properly equipped with short-range wireless interfaces and adequate storage and processing capacity, may collect information on the status of each parking spot they encounter. Moreover, they may acquire and store additional global or partial, accurate or imprecise, knowledge about the distribution of the free parking space throughout the area $A$ via communicating with other vehicles or a central server.

\footnotetext{
${ }^{1}$ Note that current navigation systems can locate parking lots, yet they cannot provide information about the availability of parking places therein.
}

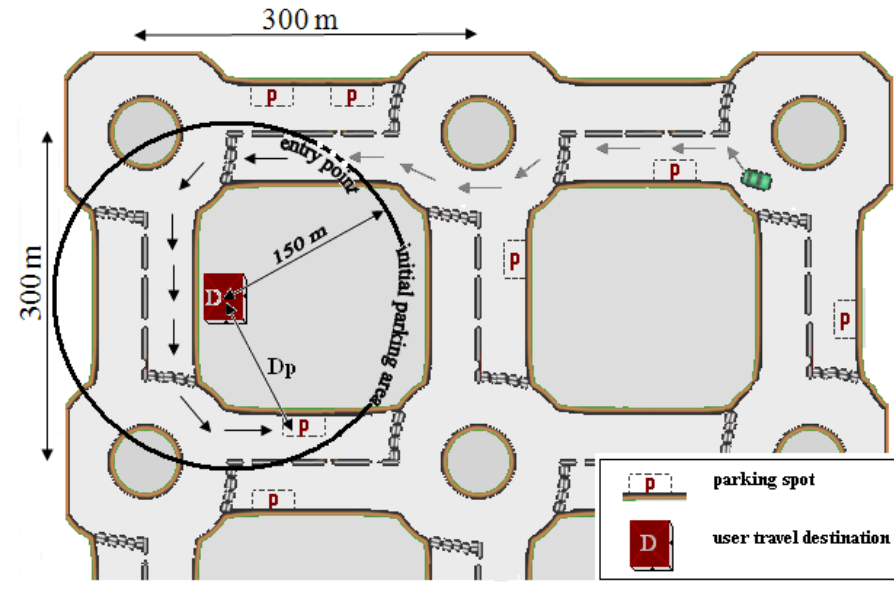

Figure 1. Section of city area $A$ showing the vehicle's path towards a parking spot close to its travel destination.

\section{A. Non-assisted parking search (NAPS)}

Usually, the parking space search process begins when the driver anticipates that he has arrived in the proximity of his travel destination (initial parking search area). This decision depends on the observed congestion level and the driver's ability to estimate the parking demand in his area of interest. Moreover, he may take into account former experience and knowledge he has acquired about the traffic flow and parking needs in the considered area.

According to the current common search strategy, drivers wander around their travel destination and sequentially check the availability of the parking spots they encounter. Typically, the radius of the search area grows progressively as parking search time increases. The search process terminates when drivers detect the first vacant parking spot in their search area. In other words, it is assumed that no one would ever reject an available parking spot located within the borders of his parking search area, under the expectation of a closer-to-destination, yet non-guaranteed, parking option.

One variant of this baseline non-assisted scheme emerges if we assume that the drivers (or vehicles) have and exploit some memory. Then, while moving around the parking search area, they could collect and store information on the location and status of each parking place they encounter and make their search more directed. In this paper, we consider the basic nonassisted parking search (NAPS) scheme as reference for our comparisons $^{2}$.

\section{B. Opportunistically-assisted parking search (OAPS)}

The information available to vehicles about the location and status of parking spots may grow with the opportunisticallyassisted parking search (OAPS) scheme. With OAPS, vehicles equipped with standard wireless interfaces such as $802.11 \mathrm{x}$ in ad hoc mode, share with each other information they acquire in the course of their search [12]. This information can be filtered in time (aging) and space, if timestamps and the geographic addresses (e.g., via GPS) of individual parking spots are stored.

\footnotetext{
${ }^{2}$ The interested reader is referred to [2] for comparisons of basic NAPS with its main variants.
} 
With such information at hand, drivers can make more informed decisions about their search. Rather than wandering randomly in the parking search area, a vehicle can now move towards the parking spot that it has stored as the closest vacant one to its destination. If it is actually vacant when it arrives at it, it occupies it. However, if the spot is occupied, he has to repeat the whole search process. In general, if a user encounters a vacant parking spot in his parking search area on his way to another selected out of his storage spot, he occupies it and terminates the search.

The opportunistic information dissemination mechanism of OAPS does not allow global common knowledge for the availability of parking space. As the status of parking spots changes in time, stored data are potentially outdated after some time interval. Therefore, the frequency of information updates is critical for the effective scheme operation. The faster information circulates across the wireless networking environment, the more accurate data will be stored in the caches of vehicles. All the same, users should be cautious when they consult their caches. Information should be filtered in order to determine the outdated information (i.e., when the timestamp of a stored record is beyond some threshold value).

On the other hand, depending on the travel destinations of users, the fast dissemination of information may synchronize the caches and, consequently, the movement patterns of individual vehicles and aggravate the effective competition for given parking spots. As we show in Section IV, how this trade off is resolved for OAPS depends on several factors such as the number of vehicles moving in the area $A$, their speed, travel preferences (destinations), and the road grid structure.

\section{Centrally-assisted parking search (CAPS)}

With CAPS, the full information processing and decisionmaking tasks lie with a central processor (server). Vehicles and parking spot sensors are only responsible for transmitting to the server parking requests and spot vacancy information, respectively. The semi real-time two-way communication of the server with the vehicles and the parking spot sensors calls for heavier network infrastructure, both wired and wireless.

When submitting its parking request, each vehicle specifies its destination to the centralized server. The server queues the requests and satisfies them in a First-Come-First-Served (FCFS) manner ${ }^{3}$, reserving for the vehicle that parking spot amongst the vacant ones, which lies closest to its destination. The user is then notified about the reservation, i.e., parking spot he should drive to. Therefore, and contrary to NAPS and OAPS, the vehicle is directed towards a guaranteed vacant parking spot. While waiting for the system assignment, the user keeps on moving towards random directions within the area.

The way the centralized system operates resembles a queuing system. In particular, when the number of vehicles $\mathrm{C}$ is finite (ref. Section III.B) and the parking time exponentially distributed, CAPS can be modelled as a finite-source G/M/P queue [13], where $\mathrm{P}$ is the number of servers (parking spots).

\footnotetext{
${ }^{3}$ Different scheduling disciplines are generally applicable when processing the parking requests. Herein, we focus on the FCFS policy for exploring the relative performance of the centralized paradigm for sharing and processing information and demonstrating the related tradeoffs.
}

The vehicles "enter the queue" when they submit a parking request and they stay there till all prior requests are served (waiting time). Their arrival process G to the "queue" depends on the time spent on driving towards a travel destination (up to the time they enter the initial parking search area). It is a function of their velocity and distance between the parking spot they have just vacated and the newly chosen destination over the road grid; both are random variables, in the generic case.

On the other hand, in assigning parking spots to vehicles, the server effectively solves an instance of the machine interference problem (MIP), also referred to as the machine repairman problem [15], with partially cross-trained repairmen. In our case, machines correspond to vehicles and parking spots to repairmen [14], who can serve more than one machine but with variable efficiency, i.e., user satisfaction according to the proximity of the spot to its travel destination.

\section{PERFormance EVAluATION METHODOlOGY}

\section{A. Performance Metrics}

When someone moves towards a specific destination, he aims for the shortest route and minimum travel time (these may not be necessarily compatible objectives). When he needs to park, on top of that, he prefers the nearest to the destination parking place (best parking place). In the ideal case, someone will reach it travelling the shortest possible route from his initial location to that parking place (best way). Therefore, the metrics we consider for comparing the three approaches to parking search are:

1) Parking search time, $T_{p s}$ : Once the driver enters the initial parking search area (Figure 1), he will start seeking for a parking place. This time is highly dependent on the parking space density in the considered area, traffic congestion level, and competition for parking space around the destination.

2) Parking search route length, $R_{p s}$ : It refers to the distance a driver travels till he parks his vehicle, measured from the moment he enters the initial parking search area. The parking space density and the demand for parking are the two factors that primarily affect $R_{p s}$ for given city area and vehicle speeds. Besides expressing user satisfaction, $R_{p s}$ and $T_{p s}$ also reflect social objectives in that more travelling results in additional fuel consumption and environmental burden.

3) Destination-parking spot distance, $D_{p}$ : It expresses the geographical distance of the two points and, contrary to $T_{p s}$ and $R_{p s}$, it is exclusively a measure of user satisfaction: the closest the parking spot lies to the destination, the more attractive it is. However, if the parking space density is small and the destination popular, the majority of users travelling towards it, will have to compromise with less desirable parking solutions.

\section{B. Simulation environment}

We have developed a simulation environment in the $\mathrm{C}$ programming language that reproduces in adequate detail the three parking search approaches. We briefly summarize it below: 
Road grid: The simulator implements a grid of two-lane roads (one lane in each direction) in a city environment; each road traverses the grid from the one side to the other, as shown in Figure 2. Additionally, there are roundabouts in every intersection, connecting up to four converging roads. Parking spots may be located in either lane of the road and are equipped with sensors providing information about their status (vacant vs. occupied).

Vehicle movement: Vehicles move within the bounds of this city environment along the horizontal and vertical roads. In each simulation time step, the next position of every vehicle depends on his current position and velocity. Every vehicle adapts its speed according to its distance from a) the front vehicle (they are not allowed to overtake one another); b) the next intersection; and c) the nearest parking spot, assuming that the car decelerates in every parking place it encounters in order to stop in a vacant one. Moreover, they zero their speed when a) they get stuck in traffic jam; b) they enter a roundabout intersection; and c) they park. Finally, the vehicles start from zero speed and are not allowed to stop or move in the reverse direction of the traffic flow.

Every time a vehicle frees a parking spot, it chooses a new destination (geographical coordinates within the bounds of the simulation area) and drives towards it. Once it reaches the destination's initial parking search area, the parking search process is initiated. Its initial parking search area is a circular area with the destination at its center and radius equal to half the distance between two adjacent intersections (Figure 2).

Under the NAPS strategy, the vehicle will circulate randomly within its initial parking search area. This random movement has been modelled by the selection of random geographical coordinates that correspond to a point inside the cyclic parking area. On the contrary, with OAPS the vehicles will filter the stored records both temporally (to exclude information that is outdated) and spatially (to consider only information about spots in the current search area).

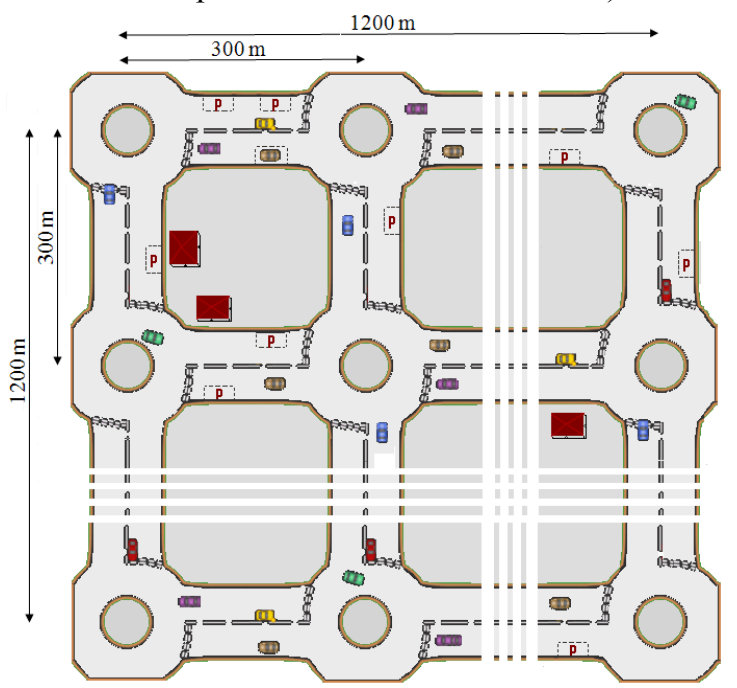

Figure 2. Simulation environment: $1200 \times 1200 \mathrm{~m}^{2}$ road grid with uniformly distributed parking places.

Out of the remaining records, the user will pick up the one that refers to the nearest-to-his-destination parking spot. If the spatiotemporal filtering step does exclude all the stored data, the user chooses randomly one spot within the parking search area and moves thereto, hoping that it may be vacated in the meanwhile. Finally, under CAPS, the user moves randomly within the parking search area till he is directed by the system to drive to the reserved parking spot.

The above procedure defines where the user should drive next. Upon arrival to this location, the user faces two possibilities: a) the location corresponds to a vacant parking spot. The user then occupies it for some time interval that may follow different distributions. When this time interval expires, he vacates the spot and selects another destination; b) the location does not correspond to a parking spot or, if it does, it is occupied - both count as failures. The user then determines whether his parking search area should grow or not. In particular, this decision will depend on the number of driver's failures in the current parking search area.

\section{Simulation set-up}

For the simulations in this paper we consider a two-lane road grid with dimensions $1200 \times 1200 \mathrm{~m}^{2}$, as shown in Figure 2. The distance between two adjacent intersections in the grid is $300 \mathrm{~m}$ and parking places are uniformly distributed alongside road lanes. The numbers of vehicles and parking spots vary to generate different parking spot densities. The mean parking time is exponentially distributed with mean equal to $1800 \mathrm{~s}$.

The duration of simulations is $10^{5} \mathrm{~s}$, which is enough time to generate a significant number of parking events in all runs. The maximum vehicle speed is set to $\mathrm{v}_{\max }=50 \mathrm{~km} / \mathrm{h}$; note that the actual instantaneous vehicle velocity may range anywhere in $\left[0, v_{\max }\right]$, as explained in Section III.B. The vehicle-parking spot sensor communication range is set to $15 \mathrm{~m}$, whereas the intra-vehicle communication range is $70 \mathrm{~m}$. In all graphs reported in Section IV, we plot the averages of ten simulation runs together with their $95 \%$ confidence intervals.

\section{Simulation Results}

We show simulation results for all three metrics presented in Section III.A. The metric values are plotted against the number of vehicles for fixed number of parking spots.

\section{A. Uniformly Distributed Destinations}

General trends: Figure 3 compares the three approaches with respect to all three metrics for a fixed number of parking spots, $\mathrm{P}=25$. Intuitively, and for all three approaches, the performance deteriorates with the number of vehicles moving in the city area $A$. Even when the travel destinations of the vehicles are uniformly spread over this area, their increase results in higher competition for individual parking spots. For NAPS and OAPS, this means that the probability to encounter a vacant spot decreases. Table 1 lists the average number of unsuccessful decisions per vehicle, i.e., how many times on average each vehicle encounters an occupied parking spot while wandering (NAPS) or driving towards a parking spot he became aware of from other vehicles (OAPS). For CAPS, there are no unsuccessful decisions; what increases is the average waiting time for the assignment of a parking spot by the central server. Moreover, the higher competition does not only 


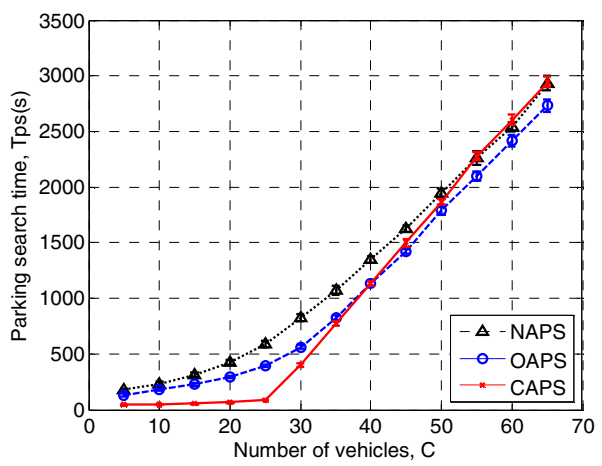

a) Parking search time

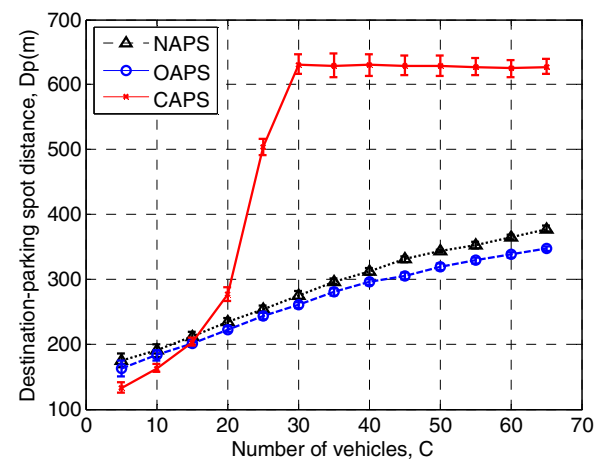

c) Destination-parking spot distance

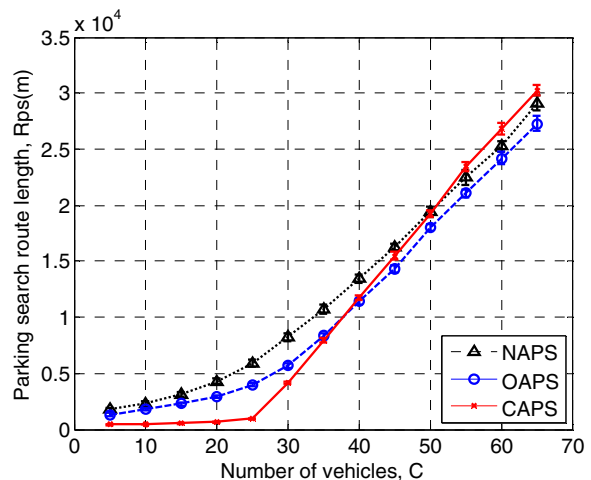

b) Parking search route length

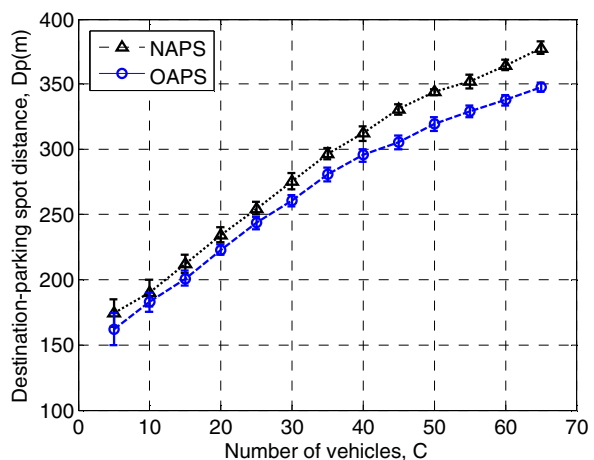

d) Destination-parking spot distance (zoom-in)

Figure 3. Comparison of the NAPS, OAPS and CAPS approaches: uniformly distributed user destinations, $\mathrm{P}=25$.

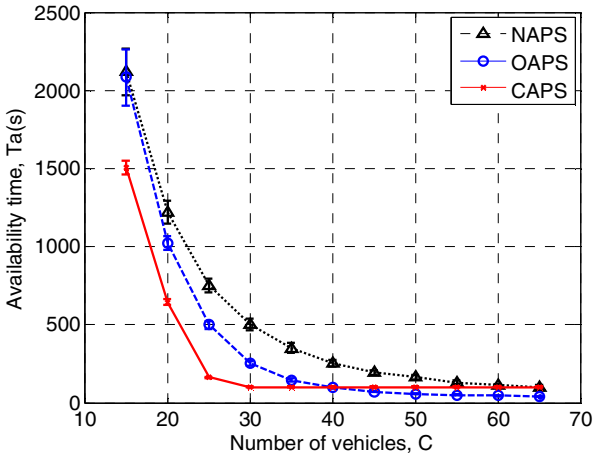

Figure 4. Average time each parking spot remains vacant: uniform distributed travel destinations, $\mathrm{P}=25$.

increase the search /waiting time and the distances that vehicles travel till they eventually park (Figure $3 \mathrm{a}$ and Figure $3 \mathrm{~b}$ ); it also results in the assignment of "worse" parking spots, located further away from the actual user travel destinations.

NAPS vs. OAPS: The benefits from information sharing and exploitation become obvious when comparing NAPS with OAPS: the opportunistic system consistently outperforms the non-assisted one for all three metrics, irrespective of the number of vehicles. With NAPS, vehicles spend much of their time wandering "blindly" without even encountering a parking spot, whether vacant or occupied. Whereas with the opportunistic system, the search is more directed and the
Table 1 Average unsuccessful parking attempts per vehicle for NAPS and OAPS: uniformly distributed travel destinations, $\mathrm{P}=25$.

\begin{tabular}{|c|c|c|c|c|c|c|}
\hline \multirow{2}{*}{$\begin{array}{c}\text { Parking } \\
\text { search } \\
\text { approach }\end{array}$} & 5 & 15 & 25 & 35 & 45 & 55 \\
\cline { 2 - 7 } & 0.27 & 1.55 & 4.33 & 9.19 & 15.18 & 21.92 \\
\hline NAPS & 0.39 & 2.39 & 6.04 & 15.03 & 26.85 & 40.22 \\
\hline OAPS & 0.39 &
\end{tabular}

Table 2 Average unsuccessful parking attempts per vehicle for NAPS and OAPS: hot-spot scenario, $\mathrm{P}=25$.

\begin{tabular}{|c|c|c|c|c|c|c|}
\hline \multirow{2}{*}{$\begin{array}{c}\text { Parking } \\
\text { search } \\
\text { approach }\end{array}$} & 5 & 15 & 25 & 35 & 45 & 55 \\
\cline { 2 - 7 } & 1.9 & 8.38 & 14.26 & 21.65 & 28.03 & 34.4 \\
\hline NAPS & 5.6 & 18.74 & 31.71 & 42.46 & 51.51 & 63.4 \\
\hline OAPS &
\end{tabular}

parking spot encounters more frequent than with OAPS. Increase of the vehicle population leads to: a) higher dissemination rates of information about parking spots amongst the vehicles. The vehicles can therefore make more informed choices as to where they should seek for (vacant) parking spots; b) more competition for the parking spots. Chances are now higher that not only the travel destinations of two or more vehicles are in close proximity but also that vehicles share the same information and, depending on their destinations, target the same parking spots. Many of the travels towards these spots prove, in the end, to be useless due to belated arrivals and only add to the total parking search time. 
Looking at Figure $3 \mathrm{a}$ and Table 1, one can see that the tradeoff faster information dissemination versus increased competition is resolved in favor of the opportunistic scheme. With OAPS, the vehicles make much better use of time than with NAPS. Within a given time window, they will discover more parking spots. Some of them will be occupied and on average, as Table 1 suggests, they will end up failing more times than in NAPS. Nevertheless, their persistent directed movement is compensated in that they manage to find vacant parking spots faster than with NAPS.

CAPS: With the centralized approach, two distinct components comprise the overall parking search time: (i) the waiting time, $T_{q}$, in the server's queue of parking requests till a vacant parking spot is found and assigned to the vehicle; and (ii) the final leg travel time, $T_{f}$, from the moment the vehicle is notified about the reserved vacant parking spot till it gets to it and occupies it. When the vehicles are fewer than or in the order of the parking spots $(\sim 30), T_{f}$ dominates the overall parking search time since there are always one or more vacant spots, where the user can be directed to. As the cars tend to outnumber parking spots, the parking requests in the server's queue start piling up and $T_{q}$ dominates the overall parking search time (Figure 6).

The second noteworthy remark about the CAPS approach is the tradeoff between the achieved parking search time (route length) and attractiveness of the assigned parking spots (Figure $3 c)$. Leaving aside very small vehicle populations, the centralized system consistently assigns parking spots that lie further away from the actual travel destinations, when compared to NAPS and OAPS. For C $>35$, all 25 parking spots are constantly reserved. Each vehicle is assigned the first place that becomes vacant, which may be located anywhere within the parking area $A$. Therefore, the average destination-parking spot distance $D_{p}$ eventually converges to the expected distance of two randomly selected points within a square area; namely, the expected value of the square line picking problem, which is known to equal $0.52 \times l=624 \mathrm{~m}$, where $l$ denotes the length of the square sides [3].

CAPS vs. OAPS and NAPS: More interesting is the way the performance ranking of the three schemes evolves. As Figure $3 \mathrm{a}$ and Figure $3 \mathrm{~b}$ suggest, their relative performance with respect to parking search time and route length changes twice. CAPS outperforms the two for $\mathrm{C}<40$, then gets worse than the opportunistic scheme and for even higher number of vehicles $(\mathrm{C}>55)$ loses to NAPS as well. The reason for this behavior is the combination of the reservation mechanism of CAPS and the more random mobility patterns of the vehicles in NAPS and OAPS.

More specifically, the better (more intensively) the systems manage to use the availability of parking spots, the better they score with respect to $T_{p s}$ and $R_{p s}$. For the centralized system, Figure 4 suggests that there is a hard bound as to how efficiently this can be done in the light of the reservation system. As the number of vehicles grows, the parking space availability drops. Eventually, they are being reserved immediately after they are released. However, a reserved spot does not necessarily accommodate a stationary vehicle. The final leg travel time, during which the vehicle drives towards the reserved parking spots, is effectively "wasted" for the system. Even worse, this time grows together with the final leg length which converges to $0.52 \times l$ for $\mathrm{C}>35$, as discussed earlier. On the contrary, both the opportunistic and, for a higher number of vehicles, the NAPS approach manage to benefit from their movement in the area and utilize almost fully the parking space availability.

In fact, the comparative performance of the systems in this scenario is an argument in favor of self-organization, and rather cooperative self-organization (OAPS).

\section{B. Hot-spot scenario}

We consider exactly the same setting with Section IV.A, only now the user travel destinations are concentrated within a particular (hotspot) road rather than being distributed uniformly over the area $\mathcal{A}$. In other words, we impose higher correlation in the mobility patterns of individual vehicles and dramatically increase the competition for certain parking spots (those located in the proximity of the hotspot).

General trends: Three are the general remarks that can directly be made when comparing the curves in Figure 5 with those in Figure 3. Firstly, the performance of all three schemes, in absolute terms, deteriorates. Secondly, the deterioration is more dramatic for the non-assisted and opportunistic schemes, whereas the centralized system experiences minimal degradation. Thirdly, and closely related to the second remark, the relative ranking of the schemes changes: contrary to what we had when user travel destinations were uniformly distributed, CAPS outperforms NAPS and OAPS throughout the vehicle population range. Moreover, the opportunistic scheme only marginally outperforms the "blind" non-assisted scheme.

NAPS and OAPS: The correlation in the location of user travel destinations does not affect all parking approaches in the same way. With NAPS, vehicles still wander "blindly", only now this wandering is bounded within a given radius around the popular road. Since the competition for a parking spot is much higher, they encounter more occupied parking spots, as can be seen from the comparison of Table 1 and Table 2. Overall, the search time and the route length increase and the vehicles need to compromise with more remote parking spots.

The synchronization artifacts are worse for the opportunistic system. With all vehicles moving in the same area, information about parking spots disseminates even faster and all vehicles end up sharing similar information. And since practically they are all interested in the same set of parking spots, the ranking of parking spots is common for all of them. Therefore, they end up following similar trajectories within the search area and often encounter occupied spots. Even worse, the information they now share is of less "value". Consider one of those vehicles competing for a vacant parking spot in the area around the popular road. The moment it finds one, it occupies it without communicating this to another vehicle. In other words, vehicles share information about where relevant parking spots are but less frequently do they become aware of vacant parking spots through information exchanges with other vehicles. Eventually, they may find a parking spot without real help from the system.

The vehicle concentration around the hotspot under NAPS and OAPS also induces congestion. As can be seen 


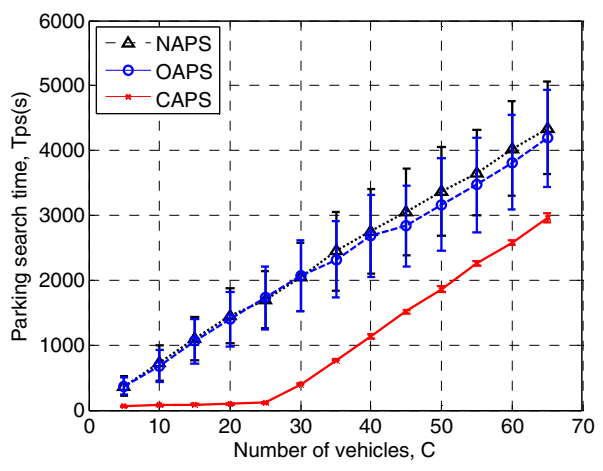

a) Parking search time

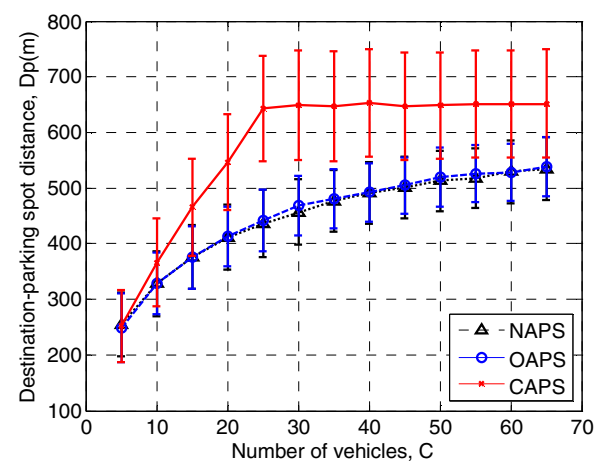

c) Destination-parking spot distance

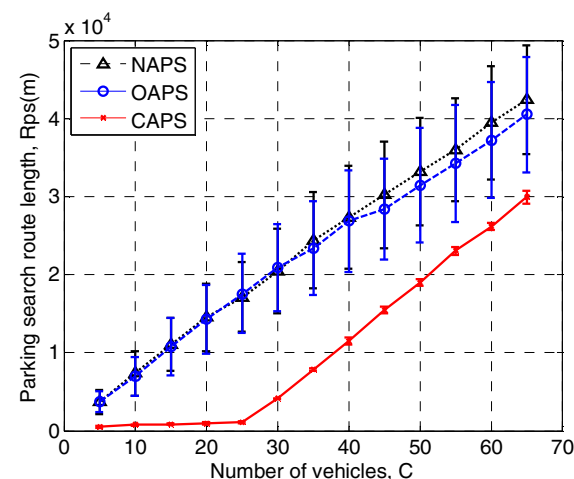

b) Parking search route length

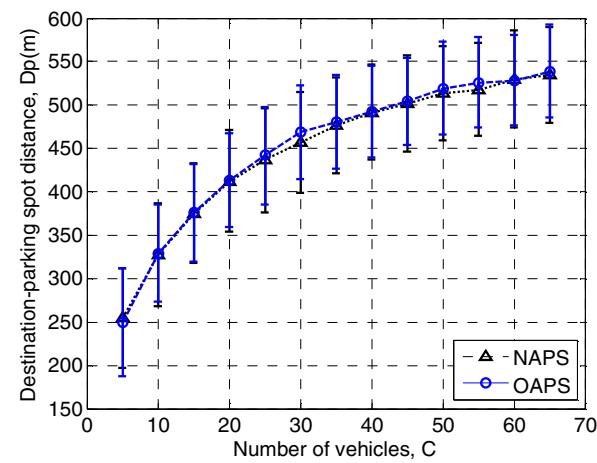

d) Destination-parking spot distance (zoom-in)

Figure 5. Comparison of the NAPS, OAPS and CAPS approaches: spatially concentrated user destinations (hot-spot scenario), $\mathrm{P}=25$.

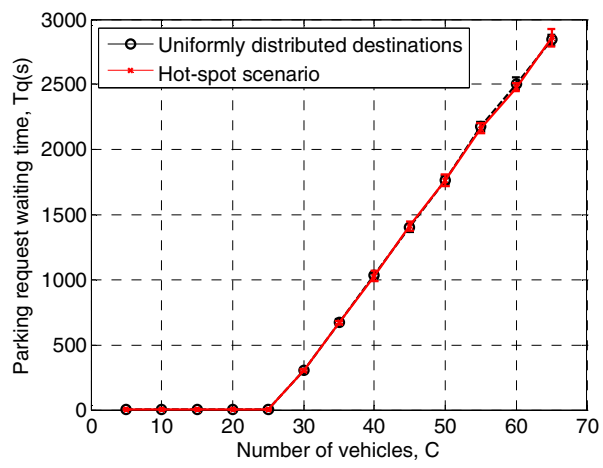

a) Parking request waiting time

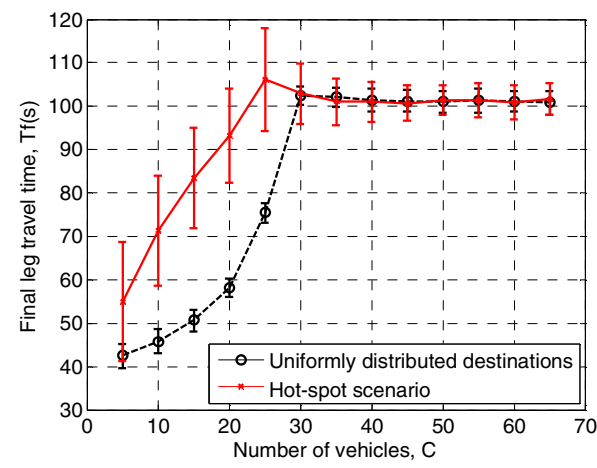

b) Final leg travel time

Figure 6. Components of the overall parking search time under CAPS: $\mathrm{P}=25$.

in Figure 5a and Figure 5b, when the vehicles grow more, the relationship between the parking search time and route length is no longer linear; vehicles break more often since they encounter more cars ahead of them (ref. Section III.B). Note that this is different than with uniformly distributed travel destinations, as Figure $3 \mathrm{a}$ and Figure $3 \mathrm{~b}$ suggest.

CAPS: The centralized approach emerges as the winning approach in the hotspot scenario. The existence of popular destinations has a different impact on the two components of the overall parking search time (see discussion in Section IV.A). The waiting time in the system queue $T_{q}$ for the parking spot assignment remains practically the same. The central server sees a similar load of parking requests, irrespective of their destinations. Contrarily to the other two approaches, having global view over the status of parking spots over the whole area $A$, it can better resolve competition amongst vehicles and make faster parking space assignments to them.
Only this comes at a penalty: the assigned parking spots lie further away from the popular road. This is why the final leg travel time, $T_{f}$, significantly exceeds its counterpart under uniformly distributed travel destinations (Figure 6). Even if for high vehicle numbers, the destination-parking spot distance converges to the same value, i.e., the expected value of the square line picking problem. Higher destination-parking spot distances emerge also for NAPS and OAPS, but the penalty is higher for the CAPS system.

\section{RELATED WORK}

As far as the academic literature is concerned, the impact of a multimode system for parking information dissemination (including real-time dissemination through the radio, and nonreal-time through newspaper advertisements and leaflets) on travelers' knowledge and decisions is assessed in [1]; whereas the impact of the adopted parking search behavior on the traffic 
flows in urban environments has been studied via an agentbased simulation model in [4]. A sophisticated system that monitors and reserves parking places within a city is introduced and shown to better distribute the traffic volume within the city in [5]. Finally, the authors in [6] present, design, implement and evaluate a system that generates a real-time map of parking space availability. The map is constructed at a central server out of aggregate parking space occupancy data, collected by vehicles circulating in the considered area.

Concerning real-world developed systems, the simplest and most common instances of parking space management are the counters placed at the entry and exit points of parking garages, which keep account of the remaining spare parking space [7]. This information is often displayed on electronic signs-boards located near parking facilities or adjacent highways. Moreover, light parking management systems have already been applied in airports and rail stations to ease passengers' parking [7][8].

An alternative proposal to the process of finding and reserving parking space in urban environments is the concept of web-based markets [9]. They use web interfaces to allow users buy and sell parking space. Drivers can reserve parking space, and owners use their privately-owned space in a profitable way. Furthermore, they offer the possibility to people occupying public on-street parking spaces to announce their estimates for the time they will be vacating them. Other online platforms present users with accurate information about the available parking places at airports and rail stations [10][11], and enable the management of reservation requests.

In our work we look into the three systems as distinct paradigms of how wireless networking solutions can leverage the power of information. For this reason, we compare three different parking search strategies that differ on the amount of information vehicles carry. Unlike the aforementioned systems, in our study, users either possess personalized parking information or collect and share parking data that they exploit independently. They neither supply any central server with parking information, nor make their parking decisions independently, exploiting a common information source.

\section{CONCLUSIONS}

We have experimentally assessed the capacity of both centralized and opportunistic wireless networking systems to assist the provision a real-world service, i.e., the parking search in city areas. Our results suggest that no parking search solution can always serve optimally the users' parking expectations. On the contrary, the particular driver preferences (e.g., travel destinations) and the density of traffic may dramatically modulate the relative capacity of the systems to collect and exploit available information for improving the service performance and give rise to tradeoffs that only the user can resolve.

Specifically, when the travel interests of users (vehicles) are randomly spread in space, the cooperative opportunistic scheme leverages the vehicle mobility and, for moderate-tohigh traffic density, can collect and disseminate fast information with broad spatial scope. The benefits from the information diffusion across the vehicles outweigh the increased competition due to overlapping interests in parking spots. On the contrary, the performance of the centralized scheme deteriorates more quickly with the traffic intensity and its reservation system appears to cancel the flexibility of more self-organizing schemes to make use of the spatially distributed parking space resource. On the contrary, when traffic concentrates in a smaller section of the area (e.g., a road), the user is faced with a harder tradeoff: either he goes for shorter parking search times and routes and parks his vehicle further away from his travel destination (centralized system); or he prefers to spend much more time and fuel in favor of a parking spot closer to his travel destination (opportunistic scheme). Notably, what he gets in the second case is marginally better than he would achieve by randomly wandering around the area (road) of interest since the information circulated by the opportunistic scheme has highly local scope and ends up leveraging the competition amongst the vehicles.

The apparent next step is to validate these experimental results against analytical models, starting from the ideas outlined in Section II.C.

\section{ACKNOWLEDGEMENTS}

This work has been supported in part by the European Commission IST-FET project RECOGNITION (FP7-IST257756) and the Marie Curie grant RETUNE (FP7-PEOPLE2009-IEF-255409).

\section{REFERENCES}

[1] A. Khattak, and J. Polak, "Effect of parking information on travelers' knowledge and behavior," Transportation, vol. 20, 1993, pp. 373-393.

[2] E. Kokolaki. On different approaches to the parking space search process. MSc Thesis, Department of Informatics and Telecommunications, University of Athens, Greece, May 2010.

[3] A.M. Mathai. An introduction to Geometrical Probability: distributed aspects with applications. Gordon \& Breaqch Science Publishers, The Netherlands, 1999.

[4] K. Dieussaert, K. Aerts, T. Steenberghen, S. Maerivoet, K. Spitaels, "SUSTAPARK: An Agent-based Model for Simulating Parking Search," in Proc. 12th AGILE International Conference on Geographic Information Science 2009 Leibniz Universität Hannover, Germany.

[5] J.L. Boehlé, L.J.M. Rothkrantz, M. van Wezel, "CBPRS: A City Based Parking and Routing System”. Research Institute of Management, Erasmus Univ. Rotterdam. ERIM Report Series ERS-2008-029-LIS.

[6] S. Mathur, T. Jin, N. Kasturirangan, J. Chandrashekharan, W. Xue, M. Gruteser and W. Trappe, "ParkNet: Drive-by Sensing of Road-Side Parking Statistics," in Proc. $8^{\text {th }}$ ACM MobiSys, San Francisco, CA, USA, June 2010.

[7] "Smart-parking at rockridge BART station, http://www.path.berkeley.edu/path/research/featured/120804/smartpark.html."

[8] R. N. Charette, "Smart Parking Systems Make It Easier to Find a Parking Space,” IEEE Spectrum Magazine, October 2007.

[9] http://www.spotscout.com.

[10] http://www.aboutairportparking.com/.

[11] http://www.parkingcarma.com.

[12] I. Carreras, I. Chlamtac, H. Woesner and H. Zhang, "Nomadic Sensor Networks," 2nd European Workshop on Wireless Sensor Networks (EWSN), January 2005.

[13] J. Sztrik, On the finite-source G/M/r queue, European Journal of Operations Research, 1985, pp. 261-268.

[14] S. Iravani, V. Krishnamurthy. Workforce agility in Repair and Mainteance Environments. Manufacturing and Service Operations Management, vol. 9, no. 2, pp. 168-184, 2007.

[15] L. Haque, M. J. Armstrong. A survey of the machine interefence problem. European Journal of Operations Research, pp. 469-482, 2007 . 